in vivo $34: 3467-3472(2020)$

doi:10.21873/invivo.12186

\title{
Dominant Antibiotic Consumption Patterns Might Be Associated With the Prevalence of Multiple Sclerosis in European Countries
}

\author{
GÁBOR TERNÁK ${ }^{1}$, KÁROLY BERÉNYI ${ }^{2}$, GERGELY MÁROVICS ${ }^{2}$, ANDRÁS SÜMEGI ${ }^{2}$, \\ BARBARA FODOR ${ }^{2}$, BALÁZS NÉMETH ${ }^{2}$ and ISTVÁN KISS ${ }^{2}$ \\ ${ }^{1}$ Medical School, Institute of Migration Health, University of Pécs, Pécs, Hungary; \\ ${ }^{2}$ Department of Public Health Medicine, University of Pécs, Pécs, Hungary
}

\begin{abstract}
Background/Aim: With a prevalence of 50-300 per 100,000 people, about 2.3 million people are estimated to live with multiple sclerosis (MS) globally. The role of antibiotics in the development, or prevention of MS is controversial. We aimed to elucidate the association between antibiotic consumption and MS. Patients and Methods: Pearson statistical comparisons were performed between the annual average antibiotic consumption patterns expressed in Defined Daily Dose/1,000 inhabitants/Day of the antibiotic consumption for the years of 1997-2018 in 30 European countries, with the respective prevalence of MS estimated for 2016. Results: A positive correlation (promoting effect) has been observed between narrow spectrum penicillin $(r=0.636)$ and tetracycline $(r=0.412)$ consumption with MS prevalence. Conclusion: Countries, with high consumption of narrow spectrum penicillin and tetracycline, experience a higher prevalence of MS than other countries.
\end{abstract}

Multiple sclerosis (MS) is a very common inflammatory neurological disease in young adults. Patients have periodic neurological relapses and the mean age of diagnosis is approximately 30 years. MS is considered as a heterogeneous disorder with variable clinical and pathological features reflecting different pathways to tissue injury. It is considered a complex disease with different clinical and pathological phenotypes, probably reflecting different pathways to tissue injury (1). The pathology of multiple sclerosis is characterized

This article is freely accessible online.

Correspondence to: Gábor Ternák, Medical School, Institute of Migration Health, University of Pécs, Pécs, Hungary. Tel: +36 72536000, Fax: +36 72501664, e-mail: gabor.ternak@aok.pte.hu

Key Words: Antibiotic consumption, multiple sclerosis, penicillin, tetracycline. by axonal or neuronal loss, demyelination, and astrocytic gliosis. Among these neuropathological characteristics, axonal or neuronal loss (referred to as neurodegeneration) is particularly relevant because it is the main underlying mechanism of permanent clinical disability (2). MS is considered primarily as an inflammatory disorder of the brain and spinal cord in which focal lymphocytic infiltration leads to damage of myelin and axons. Initially, inflammation is transient and temporal remyelination might be observed, but is not durable. The early course of disease is characterized by episodes of neurological dysfunction that usually recover. Over time the pathological changes become dominated by widespread microglial activation associated with extensive and chronic neurodegeneration, the clinical correlation of which is progressive accumulation of disability (3).

Arguments indicate that MS develops as a consequence of an autoimmune response, where effector cells from the immune system invade the patient's own brain and destroy essential tissues. This evidence comes from the particular structure of the MS lesion, from experimental animal models, and is supported by genetics and therapy (4). However, direct proof of an autoimmune cause of MS has not been yet found, as any specific autoantibody or autoreactive $\mathrm{T}$ cell directed against a self-antigen in the central nervous system can passively transfer MS to experimental animals. MS is characterized by the presence of focal demyelinated plaques within the central nervous system, accompanied by variable degrees of inflammation and gliosis, with partial preservation of axons. These lesions tend to be located in the optic nerves, spinal cord, brainstem, cerebellum and the juxtacortical and periventricular white matter. In addition, demyelinated lesions can also be found in the corpus callosum and cortical gray matter. Axonal injury can be a prominent pathologic feature of the MS plaque, though not in the acute phase (5).

Typical syndromes at appearance are of monocular visual loss due to optic neuritis, limb weakness or sensory loss due 
to transverse myelitis, double vision due to brain-stem dysfunction or ataxia due to a cerebellar lesion. After typically 10 to 20 years, a progressive clinical course develops in many of the persons affected, eventually leading to impaired mobility and cognition; approximately $15 \%$ of patients have a progressive course from onset (6). It is unlikely that multiple sclerosis will ultimately be attributed to a single cause. Rather, the genetic and environmental factor or combination of factors that result in a predisposition to multiple sclerosis, initiate the disease, and modify its course are highly diverse from one person to the next. EBV and mononucleosis, other viruses, risk genes, temperate latitude, fibrinogen, toxins, trauma, low vitamin D, smoking, obesity, early adulthood, female sex and several other factors are implicated for the development of the disease (6).

In 2016, there were 2,221,188 prevalent cases of multiple sclerosis $[95 \%$ uncertainty interval $(\mathrm{UI})=2,033,866$ $2,436,858]$ globally, which corresponded to a $10.4 \%(9.1 \%$ $11.8 \%)$ increase in the age-standardized prevalence since 1990. The highest age-standardized multiple sclerosis prevalence estimates per 100,000 population were in highincome North America (164.6; 95\% UI=153.2-177.1), Western Europe (127.0; 115.4-139.6), and Australasia (91.1; 81.5-101.7), and the lowest were in Eastern Sub-Saharan Africa (3.3; 2.9-3.8), Central Sub-Saharan African (2.8; 2.4$3.1)$, and Oceania $(2.0 ; 1.71-2.29)(7)$.

With a prevalence of 50-300 per 100000 people, about 2.3 million people are estimated to live with MS globally, although this is likely to be an underestimate given the relative lack of data from large populations including India and China (7).

Recently, there has been a substantial increase in the number of studies focusing in connecting the gut microbiome with cases of central nervous system (CNS) autoimmunity. MS is a neurodegenerative autoimmune disorder of the CNS. Recent experimental and clinical evidence suggests the presence of microbial imbalances in the gut of MS sufferers. Studies show the MS gut microbiome as having general alterations in specific taxa, some associated with the promotion of inflammatory cytokines and overall inflammation. In conjunction with these findings, experimental models of the disease have reported that $\mathrm{T}$ regulatory (Treg) cells have deficits in their function because of aberrant gut microbiota composition (8-11).

Since antibiotics are powerful agents capable of modifying the gut flora, the above observations point to the possibility that antibiotics entering humans either as therapeutic agents or from the environment are capable of triggering different, microbiome-associated diseases, including multiple sclerosis.

It was theoretically considered that if antibiotic consumption (pollution) is capable of modifying the microbiome of the intestine, the prevalence of MS might be related to the antibiotic consumption patterns in different European countries.

\section{Patients and Methods}

Based on the ECDC yearly reports of antibiotic consumption in the community from 1997 to 2018 (22 years) at ATC (Anatomical Therapeutic Chemical classification; https://www.who.int/ medicines/regulation/medicines-safety/toolkit_atc/en/) level three and level four in the group of J01C (penicillin) average yearly antibiotic consumption has been calculated for 30 European countries. The average total yearly systemic antibiotic consumption (J01) was expressed in Defined Daily Dose/1,000 inhabitants/day (DID) and the relative share of major antibiotic groups (J01 A/tetracyclin/. J01C/penicillin/. J01D/cephalosporin/. J01F/macrolide/. J01M/ quinolone/) in DID has been calculated as percentage of the total (J01) consumption. ATC level four of the penicillin group consumption (J01CE, CF, CA and CR) has been similarly counted as yearly average consumption in the years of 1997-2018.

Prevalence of Multiple Sclerosis (MS) has been calculated for 100000 inhabitants/countries calculated for 2016 in the same 30 European countries (7).

Statistical analyses. Pearson correlations has been used to calculate significance. Positive, significant correlation was estimated, when the $r$ value was a positive number and the significance $(p)$ was $\leq 0.05$. Negative significance was considered, when the $r$ value was a negative number and similarly, the $p$-value was $\leq 0.05$.

\section{Results}

Comparisons were made between antibiotic consumption data and country prevalence of MS for 100,000 inhabitants in 30 European countries included in the study. Rank order of the MS prevalence in the countries is shown in Table I. The highest numbers were observed in Sweden and Denmark (201.52/100,000 and 201.85/100,000).

According to the ECDC yearly reports of antibiotic consumption in the community from 1997 to 2018 at ATC level three and level four in the group of J01C (penicillin) average yearly antibiotic consumption has been also calculated for 30 European countries (Table II). A strong positive correlation has been observed with tetracycline (J01A) (Pearson: $r=0.412, p=0.024)$ and narrow spectrum penicillin consumption ( $01 \mathrm{CE}+\mathrm{CF}$ ) (Pearson: $r=0.636$, $p<0.001$ ) (Figure 1) and a strong negative association with broad spectrum combination penicillin (J01CR) (Pearson: $r=-0.528, p=0.003$ ), cephalosporin (J01D) (Pearson: $r=-$ $0.603, p<0.001)$ and quinolone (J01M) (Pearson: $r=-0.576$, $p=0.001$ ) (Figure 2).

Our study clearly indicates the role of certain antibiotics (tetracycline, narrow spectrum penicillin) in the development of MS probably acting through the modification of gut flora. This process might be inhibited by broad spectrum combination penicillin (J01CR) and other broad-spectrum antibiotics, like cephalosporin (J01D) and quinolone (J01M), while macrolides $(\mathrm{J} 01 \mathrm{~F})$ did not show any supportive or protective association with the prevalence of MS. It is of importance that broad spectrum beta-lactamase sensitive 
Ternák et al: Antibiotic Consumption Patterns and the Prevalence of Multiple Sclerosis

Table I. Rank order (decreasing) of prevalence of multiple sclerosis calculated for 100,000 inhabitants/countries (2016) and rank order (decreasing) of the consumption of antibiotics with "promoting" effect on the prevalence of MS.

\begin{tabular}{|c|c|c|c|c|c|}
\hline Countries & $\begin{array}{c}\text { Multiple sclerosis } \\
\text { prevalence/100,000 } \\
\text { population }(2016)\end{array}$ & Countries & $\begin{array}{l}\text { Relative share in \% of } \\
\text { MS "enhancing" antibiotics } \\
\text { (J01A+CE+CF) within } \\
\text { the global consumption } \\
\text { (average 1997-2018) }\end{array}$ & Countries & $\begin{array}{l}\text { Relative share in } \% \text { of } \\
\text { MS "inhibitory" antibiotics } \\
\text { (J01CR+J01D+J01M) within } \\
\text { the global consumption } \\
\text { (average 1997-2018) }\end{array}$ \\
\hline
\end{tabular}

\begin{tabular}{|c|c|c|c|c|c|}
\hline Denmark & 201.85 & Sweden & 60.21 & Norway & 4.43 \\
\hline Sweden & 201.52 & Denmark & 50.87 & Denmark & 6.09 \\
\hline Ireland & 163.66 & Norway & 46.96 & Sweden & 9.84 \\
\hline UK & 157.09 & Iceland & 44.40 & UK & 12.54 \\
\hline Finland & 148.25 & UK & 37.79 & Iceland & 18.34 \\
\hline Netherlands & 147.18 & Finland & 33.51 & Netherlands & 20.22 \\
\hline Iceland & 146.47 & Netherlands & 33.29 & Finland & 23.66 \\
\hline Norway & 139.14 & Germany & 29.60 & Lithuania & 24.20 \\
\hline Luxembourg & 135.85 & Czech Rep. & 28.31 & Latvia & 25.27 \\
\hline Germany & 133.69 & Estonia & 23.30 & Estonia & 26.49 \\
\hline Belgium & 127.46 & Latvia & 23.10 & Czech Rep. & 28.68 \\
\hline Austria & 123.1 & Slovakia & 22.10 & Germany & 29.14 \\
\hline Italy & 119.58 & Slovenia & 20.82 & Poland & 31.63 \\
\hline France & 100.39 & Lithuania & 18.64 & Ireland & 32.38 \\
\hline Poland & 95.2 & Bulgaria & 18.50 & Bulgaria & 34.89 \\
\hline Lithuania & 95.02 & Austria & 17.51 & France & 34.97 \\
\hline Spain & 93.8 & Poland & 16.78 & Slovenia & 35.50 \\
\hline Portugal & 81.96 & Hungary & 15.27 & Slovakia & 40.94 \\
\hline Greece & 73.98 & France & 15.21 & Belgium & 42.95 \\
\hline Slovenia & 71.52 & Ireland & 13.84 & Austria & 45.39 \\
\hline Hungary & 69.08 & Croatia & 13.79 & Greece & 46.30 \\
\hline Czech Rep. & 68.97 & Belgium & 12.93 & Hungary & 48.01 \\
\hline Latvia & 68.83 & Cyprus & 12.19 & Croatia & 48.77 \\
\hline Estonia & 68.8 & Luxembourg & 11.23 & Luxembourg & 49.63 \\
\hline Slovakia & 61.77 & Italy & 10.77 & Italy & 52.97 \\
\hline Malta & 51.7 & Greece & 9.89 & Spain & 53.49 \\
\hline Cyprus & 50.63 & Romania & 9.81 & Portugal & 54.59 \\
\hline Croatia & 49.11 & Portugal & 9.11 & Romania & 55.28 \\
\hline Bulgaria & 44.69 & Spain & 7.65 & Cyprus & 61.38 \\
\hline Romania & 43.74 & Malta & 7.18 & Malta & 65.05 \\
\hline
\end{tabular}

Tetracyclin: J01A. Narrow spectrum penicillinase sensitive penicillin: J01CE. Narrow spectrum penicillinase resistant penicillin: J01CF; and the rank order (increasing) of the consumption of antibiotics with "protective" effect on the prevalence of MS (broad spectrum penicillin combined with beta-lactamase inhibitors: J01CR, cephalosporins: J01D, quinole: J01M). The upper third of the countries (10 countries) with the highest prevalence rate of MS can be found among the highest consumers of the "promoting" group of antibiotics (eight out of ten countries) and among the lowest consumers of the "inhibitory" group of antibiotics (seven out of ten countries).

penicillin alone (J01CA) did not show any promoting or inhibitory effect on the prevalence of MS, only if it was combined with beta-lactamase inhibitors (clavulic acid, sulbactam). This observation calls the possible inhibitory (protective) effect of beta-lactamase inhibitors, which might deserve further consideration and possible research as a therapeutic agent for MS.

The role of antibiotics in the development, or prevention of MS is controversial. Large-scale case control studies indicated a reduced number of MS in the group of population taking antibiotics compared to the control group (12). In another study more than 2 weeks of penicillin use was associated with a $50 \%$ reduction in the risk of MS (13).
Broad and narrow spectrum penicillin were not separately calculated (J01CE, J01CF, J01CA, J01CR) in this study. Based on our observations, it could be suspected that mostly broad spectrum-combination penicillin was consumed by the patients (J01CR) included in the study, which showed a strong negative association with the prevalence of MS. In animal models, the animals (rats), treated with minocycline did not develop neurologic dysfunction nor had a less severe course than untreated rats. The drug was tested in rats with autoimmune encephalomyelitis. In a small scale clinical trial $100 \mathrm{mg}$ of minocycline, administered orally twice daily, was observed to delay the conversion of the clinically isolated demyelination syndrome to multiple sclerosis, as defined 
in vivo $34: 3467-3472(2020)$

Table II. Yearly, average antibiotic consumption, calculated from the annual antibiotic consumption report of the ECDC (1997-2018).

\begin{tabular}{|c|c|c|c|c|c|c|c|c|c|c|c|c|}
\hline $\begin{array}{l}\text { ECDC } \\
1997-2018\end{array}$ & $\begin{array}{c}\mathrm{J} 01 \\
100 \%\end{array}$ & $\begin{array}{c}\mathrm{J} 01 \mathrm{~A} \\
\%\end{array}$ & $\begin{array}{c}\mathrm{J} 01 \mathrm{C} \\
\%\end{array}$ & $\begin{array}{c}\text { J01CA } \\
\%\end{array}$ & $\begin{array}{c}\mathrm{J} 01 \mathrm{CR} \\
\%\end{array}$ & $\begin{array}{c}\mathrm{J} 01 \mathrm{CA}+\mathrm{CR} \\
\%\end{array}$ & $\begin{array}{c}\text { J01CE } \\
\%\end{array}$ & $\begin{array}{c}\mathrm{J} 01 \mathrm{CF} \\
\%\end{array}$ & $\begin{array}{c}\mathrm{J} 01 \mathrm{CE}+\mathrm{CF} \\
\%\end{array}$ & $\begin{array}{c}\mathrm{J} 01 \mathrm{D} \\
\%\end{array}$ & $\begin{array}{c}\mathrm{J} 01 \mathrm{~F} \\
\%\end{array}$ & $\begin{array}{c}\mathrm{J} 01 \mathrm{M} \\
\%\end{array}$ \\
\hline Austria & 12.12 & 9.21 & 35.74 & 6.92 & 20.7 & 27.54 & 8.23 & 0.07 & 8.30 & 13.14 & 26.71 & 11.56 \\
\hline Belgium & 21.96 & 11.33 & 40.06 & 17.3 & 21.03 & 38.31 & 0.44 & 1.17 & 1.60 & 11.12 & 14.63 & 10.79 \\
\hline Bulgaria & 17.38 & 13.34 & 37.44 & 23.45 & 9 & 32.46 & 5.07 & 0.10 & 5.16 & 14.71 & 14.28 & 11.18 \\
\hline Croatia & 18.59 & 7.9 & 42.18 & 13.58 & 22.7 & 36.28 & 5.73 & 0.16 & 5.90 & 17.72 & 15.06 & 8.35 \\
\hline Cyprus & 26.94 & 11.76 & 35.17 & 11.34 & 23.58 & 34.93 & 0.34 & 0.09 & 0.43 & 21.53 & 11.61 & 16.27 \\
\hline Czech Rep. & 15.01 & 15.12 & 36.71 & 6.66 & 13.03 & 22.36 & 12.76 & 0.43 & 13.19 & 8.29 & 19.89 & 7.36 \\
\hline Denmark & 14.18 & 9.84 & 62.58 & 19.01 & 2.51 & 21.34 & 33.53 & 7.50 & 41.03 & 0.20 & 14.90 & 3.37 \\
\hline Estonia & 10.4 & 20.8 & 32.87 & 20.29 & 9 & 29.3 & 2.44 & 0.06 & 2.50 & 9.42 & 19.39 & 8.07 \\
\hline Finland & 16.79 & 23.89 & 29.75 & 15.87 & 4.5 & 20.37 & 9.23 & 0.40 & 9.62 & 13.85 & 10.35 & 5.31 \\
\hline France & 24.87 & 13.04 & 44.12 & 26.85 & 15.74 & 41.6 & 0.72 & 1.46 & 2.17 & 11.37 & 16.61 & 7.87 \\
\hline Germany & 12.89 & 20.99 & 27.38 & 16.65 & 2.38 & 19.03 & 8.50 & 0.11 & 8.61 & 16.82 & 19.02 & 9.94 \\
\hline Greece & 30.42 & 8.26 & 27.81 & 13.44 & 12.79 & 26.17 & 1.62 & 0.01 & 1.63 & 24.59 & 27.75 & 8.92 \\
\hline Hungary & 14.96 & 10.68 & 35.96 & 10.26 & 21.1 & 31.36 & 4.59 & 0.00 & 4.59 & 14.28 & 20.77 & 12.64 \\
\hline Iceland* & 19.47 & 25.46 & 48.22 & 17.47 & 11.58 & 29.06 & 12.99 & 5.95 & 18.94 & 2.57 & 8.19 & 4.19 \\
\hline Ireland & 18.25 & 2.8 & 44.97 & 14.84 & 18.88 & 33.73 & 5.05 & 5.98 & 11.04 & 8.49 & 18.90 & 5.01 \\
\hline Italy & 22 & 10.65 & 42.44 & 16.45 & 25.53 & 41.99 & 0.05 & 0.07 & 0.12 & 12.70 & 21.78 & 14.75 \\
\hline Latvia & 10.58 & 22.15 & 38.09 & 26.4 & 10.9 & 37.31 & 0.93 & 0.02 & 0.95 & 4.87 & 13.10 & 9.50 \\
\hline Lithuania & 15.88 & 10.6 & 48.13 & 31.75 & 8.46 & 40.22 & 7.63 & 0.41 & 8.04 & 9.00 & 12.04 & 6.74 \\
\hline Luxembourg & 23 & 9.94 & 34.96 & 13.42 & 20.04 & 33.33 & 0.42 & 0.87 & 1.29 & 18.62 & 18.17 & 10.96 \\
\hline Malta & 18.83 & 6.53 & 33.49 & 3.15 & 30.26 & 33.42 & 0.39 & 0.27 & 0.66 & 23.24 & 20.58 & 11.55 \\
\hline Netherlands & 9.341 & 25.57 & 32.11 & 13.72 & 10.44 & 24.17 & 4.06 & 3.65 & 7.72 & 0.60 & 14.74 & 9.18 \\
\hline Norway & 15.29 & 19.38 & 40.57 & 12.83 & 0 & 12.68 & 24.16 & 3.42 & 27.58 & 1.02 & 10.92 & 3.42 \\
\hline Poland & 18.77 & 14.46 & 33.69 & 20.94 & 12.03 & 32.33 & 2.21 & 0.11 & 2.32 & 12.78 & 18.23 & 6.82 \\
\hline Portugal & 18.34 & 5.74 & 42.35 & 11.9 & 27.67 & 39.57 & 0.16 & 3.21 & 3.38 & 12.67 & 17.48 & 14.25 \\
\hline Romania & 24.14 & 4.1 & 47.17 & 18.12 & 23.19 & 41.32 & 3.10 & 2.60 & 5.71 & 19.03 & 11.51 & 13.06 \\
\hline Slovakia & 21.51 & 8.56 & 39.4 & 10.5 & 15.14 & 25.62 & 13.46 & 0.08 & 13.54 & 16.87 & 22.06 & 8.93 \\
\hline Slovenia & 13.18 & 4.06 & 55.39 & 16.6 & 21.78 & 23.52 & 15.66 & 1.10 & 16.76 & 3.83 & 19.39 & 9.89 \\
\hline Spain & 17.26 & 5.79 & 51.1 & 20.62 & 28.44 & 49.07 & 0.58 & 1.28 & 1.86 & 11.25 & 14.70 & 13.80 \\
\hline Sweden & 13.8 & 22.01 & 47.44 & 7.59 & 1.3 & 8.17 & 28.68 & 9.53 & 38.21 & 2.32 & 5.50 & 6.23 \\
\hline UK & 15.25 & 25.78 & 38.39 & 21.37 & 4.95 & 26.32 & 4.86 & 7.15 & 12.01 & 3.97 & 17.30 & 3.62 \\
\hline
\end{tabular}

J01: Total consumption of systemic antibiotics in Defined Daily Dose/1,000 inhabitants/day (DID). J01A: tetracycline. J01C: penicillin. J01CA: Broad spectrum, beta-lactamase sensitive penicillin. J01CR: Broad spectrum, beta-lactamase combination penicillin. J01CE: Narrow spectrum, betalactamase sensitive penicillin. J01CF: Narrow spectrum, beta-lactamase resistant penicillin. J01D: Cephalosporin. J01F: Macrolide. J01M: Quinolone, calculated as relative share in $\%$ of the $\mathrm{J} 01 . *$ Country provided only total care data.

according to the 2005 McDonald criteria at 6 months followup, but at 12 months, no difference was observed compared to the placebo group (14).

\section{Conclusion}

Our study clearly supports the existence of the long-suspected relationship between antibiotics, microbiome and the prevalence of MS. It is of importance to stress that when discussing the effect of antibiotics on the microbiome and the possible related ailments (obesity, diabetes, etc., or Parkinson's diseases as we published before) (15), different antibiotic molecules effecting different microbial taxa and they might have different either protective or promoting effects on related disorders. Our present paper indicates a similar antibiotic consumption pattern in MS in countries (mostly Scandinavian) with high narrow spectrum penicillin and tetracycline consumption. Regarding our results, it might be suspected that certain antibiotics (tetracycline, narrow spectrum penicillin) facilitate the production of so called "enhancer molecule" generating microbial taxa promoting the development of MS through inflammatory, autoimmune mechanisms. Other antibiotics (broad-spectrum combination penicillin, cephalosporin, quinolone) might promote the production of "protective molecules" generated by other microbial taxa, reducing the inflammatory/immune mechanisms leading to the development of MS. It might be of importance that broad spectrum, beta lactamase-sensitive (J01CA) penicillin did not show any significant association with the prevalence of MS, while the broad-spectrum beta lactamase combination penicillin (J01CR) has strong protective activity, indicating the possible effect of beta lactamases. 
- MS prev./ 100,000

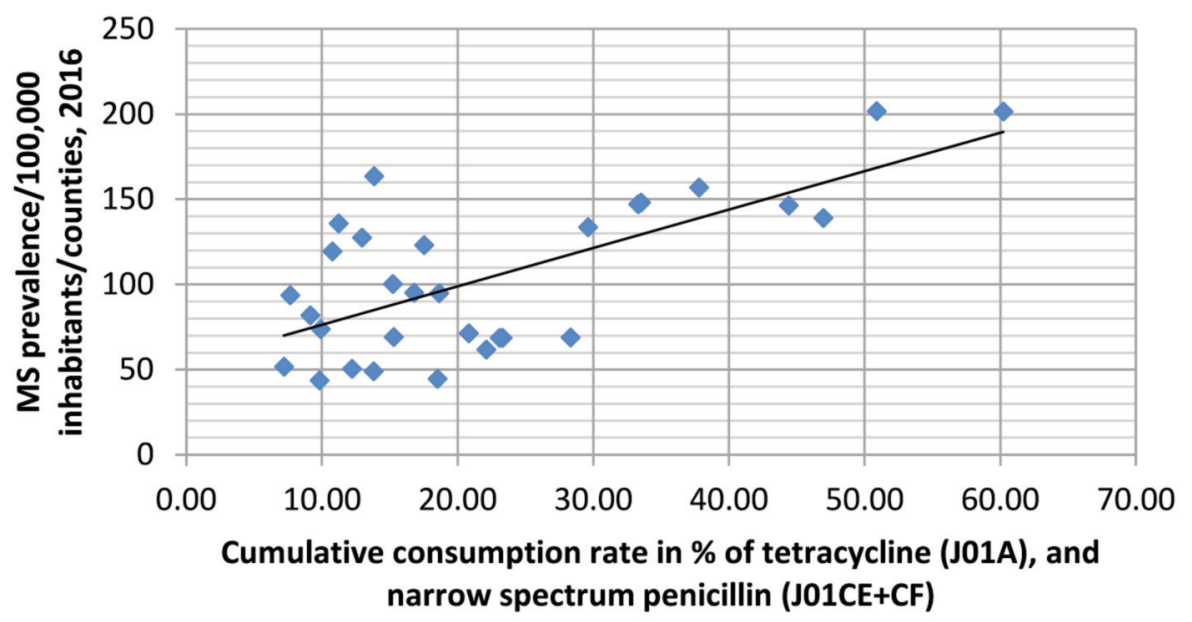

Figure 1. Positive, significant association between tetracyclin (J01A) plus narrow spectrum penicillin (J01CE+CF) and the prevalence of multiple sclerosis $(M S)$.

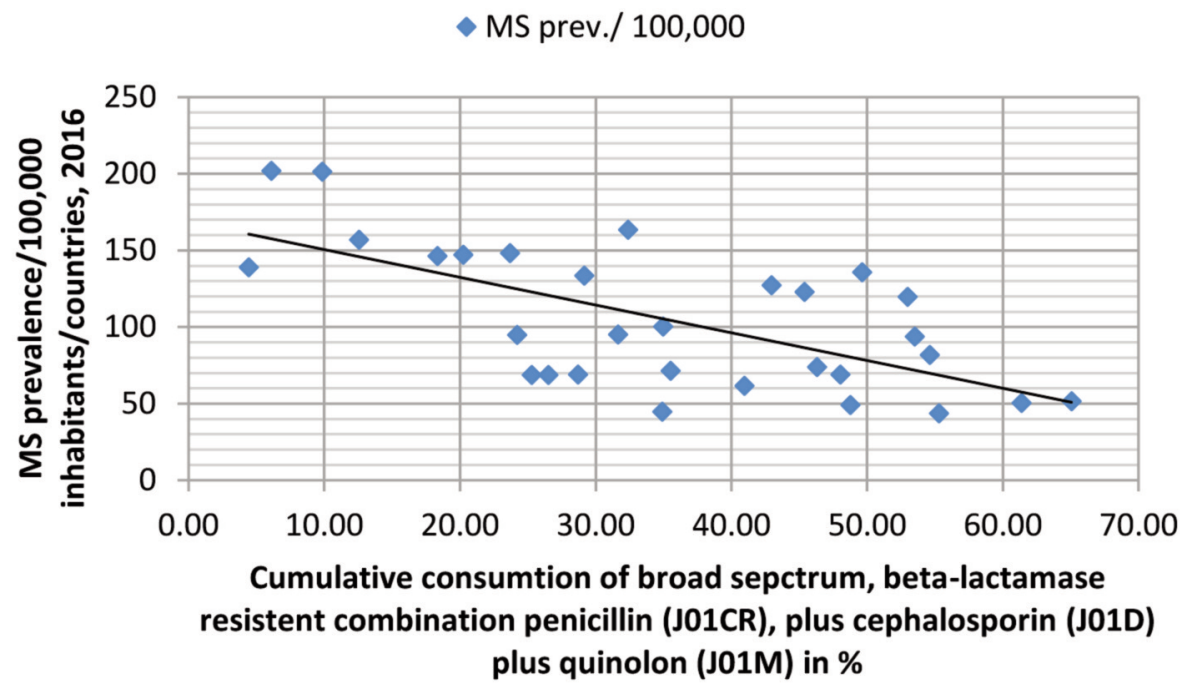

Figure 2. Negative (protective) association between multiple sclerosis (MS) prevalence and the cumulative consumption share of the total, systemic antibiotic consumption (J01) of broad spectrum, beta lactamase-resistant, combination penicillin (J01CR), plus cephalosporin (J01D), quinolone (J01M) in \%.

As this was a retrospective statistical analysis, it could not be proofed at individual level that the utilization of "promoting" and "protective" antibiotics shows a direct effect on the progression or amelioration the symptoms of MS. The role of other confounding factors could not be analyzed in this study, due to the lack of individual data, and hence the possible modification effect of such circumstances could not be taken into consideration.

Using large databases, it might be strongly suspected that antibiotics exhibit either "promoting" or "protective" effect on the development of MS through the modification of gut flora. This concept is being supported by the observation that countries with high consumption patterns of narrow spectrum penicillin and tetracycline and low consumption of broadspectrum combination penicillin, cephalosporin and quinolone (mostly Scandinavian countries), showed a higher prevalence rate of MS than others (mostly Mediterranean countries). The possible protective role of beta lactamases (mostly clavulic acid) in the development of MS is identified and might be utilized as a therapeutic agent to treat MS. 


\section{Conflicts of Interest}

The Authors have no conflicts of interest to declare.

\section{Authors' Contributions}

GT: Developed the concept, calculated the data sheets, has written the manuscript. KB: Calculated and compared the data bases, corrected data when it seemed to be necessary. GM: Searching of the literature. AS: Developed tables of datasheets. BF: Searched the literature, created figures. BN: Developed the outline of the manuscript and corrected the structure of the text, proposed alterations, when it was necessary. IK: Reviewed the manuscript, searched and proposed the appropriate literature included in the manuscript.

\section{References}

1 Weiner HL: Multiple sclerosis is an inflammatory T-cellmediated autoimmune disease. Arch Neurol 61(10): 1613-1615, 2004. PMID: 15477521 DOI: 10.1001/archneur.61.10.1613

2 Thompson AJ, Baranzini SE, Geurts J, Hemmer B and Ciccarelli: Lancet 391: 1622-1636, 2018. PMID: 29576504. DOI: $10.1016 / \mathrm{S} 0140-6736(18) 30481-1$

3 Compston A and Coles A: Multiple sclerosis. Lancet 372(9648): 1502-1517, 2008. PMID: 18970977. DOI: 10.1016/S01406736(08)61620-7

4 Wekerle H: Nature plus nurture: the triggering of multiple sclerosis. Swiss Med Wkly 145: w14189, 2015. PMID: 26430854. DOI: $10.4414 / \mathrm{smw} .2015 .14189$

5 Goodin DS: Pathogenesis of multiple sclerosis. Clin Exp Neuroimmunol 6(Suppl 1): 2-22, 2015. DOI: 10.1111/cen3. 12261

6 Reich DS, Lucchinetti CF and Calabresi PA: Multiple sclerosis. N Engl J Med 378: 169-180, 2018. PMID: 29320652. DOI: 10.1056/NEJMra1401483

7 GBD 2016 Multiple Sclerosis Collaborators: Global, regional and national burden of multiple sclerosis 1990-2016: a systematic analysis for the Global Burden of Disease Study 2016. Lancet Neurol 18: 269-285, 2019. PMID: 30679040. DOI: 10.1016/S1474-4422(18)30443-5

8 Kirby TO and Ochoa-Repáraz J: The Gut microbiome in multiple sclerosis: A potential therapeutic avenue. Med Sci 6: 69, 2018. PMID: 30149548. DOI: 10.3390/medsci6030069
9 Chu F, Shi M, Lang Y, Shen D, Jin T, Zhu J and Cui L: Gut microbiota in multiple sclerosis and experimental autoimmune encephalomyelitis: Current applications and future perspectives. Mediators Inflamm 2018: 8168717, 2018. PMID: 29805314. DOI: $10.1155 / 2018 / 8168717$

10 Berer K, Gerdes LA, Cekanaviciute E, Jia X, Xiao L, Xia Z, Liu C, Klotz L, Stauffer U, Baranzini SE, Kümpfel T, Hohlfeld R, Krishnamoorthy G and Wekerle H: Gut microbiota from multiple sclerosis patients enables spontaneous autoimmune encephalomyelitis in mice. Proc Natl Acad Sci USA 114(40): 10719-10724, 2017. PMID: 28893994. DOI: 10.1073/pnas. 1711233114

11 Tremlett H, Fadrosh DW, Faruqi AA, Zhu F, Hart J, Roalstad S, Graves J, Lynch S, Waubant E and US Network of Pediatric MS Centers: Gut microbiota in early pediatric multiple sclerosis: a case-control study. Eur J Neurol 23: 1308-1312, 2016. PMID: 27176462. DOI: $10.1111 /$ ene. 13026

12 Abdollahpour I, Nedjat S, Mansournia MA, Eckert S and Weinstock-Guttman B: Infectious exposure. antibiotic use. and multiple sclerosis: A population-based incident case-control study. Acta Neurol Scand 138: 308-314, 2018. PMID: 29740825. DOI: 10.1111/ane.12958

13 Alonso A, Jick SS, Jick H and Hernán AM: Antibiotic use and risk of multiple sclerosis. Am J Epidemiol 163: 997-1002, 2006. PMID: 16597708. DOI: 10.1093/aje/kwj123

14 Metz LM, Li DKB, Traboulsee AL, Duquette P, Eliasziw M, Cerchiaro G, Greenfield J, Riddehough A, Yeung M, Kremenchutzky M, Vorobeychik G, Freedman MS, Bhan V, Blevins G, Marriott JJ, Grand'Maison F, Lee L, Thibault M, Hill MD and Yong VW: Trial of minocycline in a clinically isolated syndrome of multiple sclerosis. N Engl J Med 376: 2122-2133, 2017. PMID: 28564557. DOI: 10.1056/NEJMoa1608889

15 Ternák G, Kuti D and Kovács K: Dysbiosis in Parkinson's disease might be triggered by certain antibiotics. Med Hypotheses 137: 109564, 2020. DOI: 10.1016/j.mehy.2020.109564 